\title{
Liquid liothyronine to obtain target TSH in differentiated thyroid cancer patients
}

\author{
Pierpaolo Trimboli ${ }^{1), 2)}$, Marco Centanni ${ }^{3), 4)}$ and Camilla Virili ${ }^{3), 4)}$ \\ 1) Department of Nuclear Medicine and Thyroid Centre, Oncology Institute of Southern Switzerland, Bellinzona, Switzerland \\ 2) Section of Endocrinology and Diabetology, Ospedale Israelitico, Rome, Italy \\ 3) Department of Medico-surgical Sciences and Biotechnologies, Sapienza University of Rome, Latina, Italy \\ 4) UOC Endocrinology, AUSL Latina, Latina, Italy
}

\begin{abstract}
In the last ten years a liquid formulation of liothyronine (L-T3) became available. To date, no studies on its systematic use have been reported. This study is aimed at assessing the reliability of liquid L-T3 in achieving target TSH in patients with differentiated thyroid cancers (DTC). Twenty-one high risk DTC patients in whom levothyroxine treatment up to $2.0 \mu \mathrm{g} / \mathrm{kg}$ /day did not suppress TSH levels (i.e. $>0.1 \mathrm{mIU} / \mathrm{L}$ ) were selected. Maintaining the same L-T4 dose, they started to assume liquid L-T3 at an initial fixed dose of $3.55 \mu \mathrm{g}$ ( 5 drops). Further adjustments of L-T3 dose were tailored according to individual assessment. Initial serum TSH ranged from 0.8 to $12.0 \mathrm{mIU} / \mathrm{L}$, when patients assumed high dose of L-T4 alone. Following the addition of a daily single dose of $3.55 \mu \mathrm{g}$ L-T3, the target TSH was attained in five patients (23.8\%). After increasing L-T3 dose up to a mean of $7.3 \pm 3.4 \mu \mathrm{g} /$ day all patients reached target serum TSH $(<0.1 \mathrm{mIU} / \mathrm{L})$. The mean individual L-T3 dose was significantly correlated with the body weight and was $0.11 \pm 0.04 \mu \mathrm{g} / \mathrm{kg} / \mathrm{day}(p=0.013)$. Mean L-T4:L-T3 ratio was 21:1. No patients showed skewed free- $\mathrm{T}_{3}$ or free- $\mathrm{T}_{4}$ values, neither experienced discomfort nor reported adverse events. Liquid L-T3 can be useful to achieve optimal TSH suppression in high risk DTC with not suppressed TSH on L-T4 alone. This formulation allows an individual tailoring of L-T3, minimizing risks of side effects as well as of overtreatment in these clinical conditions.
\end{abstract}

Key words: Thyroid cancer, Liothyronine, Therapy, Hypothyroidism

THYROID neoplasia is a common entity largely represented by differentiated thyroid carcinoma (DTC), mostly papillary. The initial treatment of these patients includes surgical excision of thyroid gland and, in specific cases, neck lymph nodes dissection. Subsequently, patients may undergo radioiodine treatment $\left({ }^{131} \mathrm{I}\right)$ [1]. After this initial approach, DTC patients take thyroid hormone therapy to replace thyroid gland production and suppress TSH levels, when indicated. Serum TSH suppression is specifically recommended in those patients with advanced or more aggressive cancer type who can be stratified as at high risk for recurrence/ persistence of disease [1-3]. The way to achieve the desired serum levels of TSH in different thyroid disorders is, as yet, a debated issue [4]. Traditionally,

Submitted Jan. 22, 2016; Accepted Mar. 8, 2016 as EJ16-0040 Released online in J-STAGE as advance publication Mar. 18, 2016 Correspondence to: Pierpaolo Trimboli, Department of Nuclear Medicine and Thyroid Centre, Oncology Institute of Southern Switzerland, Via Ospedale 12, Bellinzona 6500, Switzerland. E-mail: pierpaolo.trimboli@gmail.com

(C) The Japan Endocrine Society levothyroxine (L-T4) alone has been considered the treatment of choice in athyreotic subjects, sometimes with a dose higher than that one would expect [5]. To obtain full suppression of TSH levels (i.e. $<0.1 \mathrm{mIU} / \mathrm{L}$ ) the dose of L-T4 may be high enough to cause several adverse effects in this long lasting treatment [6]. In these athyreotic subjects the problem has been faced by using a combined L-T4 and liothyronine (L-T3) treatment [7]. Rationale for this treatment was that thyroid gland secretes about $20 \%$ of circulating triiodothyronine to a final serum thyroxine/triiodothyronine ratio up to $15: 1$ [8]. Following thyroidectomy, the lack of thyroid contribution to circulating triiodothyronine might impair the whole thyroid hormones homeostasis and a undefined number of athyreotic patients might not reach this novel balance [9]. Several studies dealt with the exact proportion between L-T3 and L-T4 dose highlighting also the occurrence of side effects [10]. In fact, the specific ETA guidelines on this subject recommend to use much lower doses of L-T3 to enhance safety; despite these efforts, no agreement has been 
reached on the different L-T4/L-T3 proportion nor on the administration's schedule. So far, a consensus of L-T4 plus L-T3 treatment has not been achieved and the use of L-T3 is currently not recommended [10].

In the last decade a liquid formulation of L-T3 (IBSA, Switzerland) has been made available in several countries. This product could allow physicians to administrate a tailored L-T3 dose and obtain the target TSH value, avoiding potential side effects due to overtreatment. To date, no studies on the systematic use of liquid L-T3 in DTC patients have been reported. Also, in Italy, the tablet formulation of L-T3 is commercialized in a single dose of $20 \mu \mathrm{g}$ and recently is discontinuously available.

The aim of our study was to evaluate, with a prospective design, the reliability of liquid L-T3 treatment in achieving the optimal TSH values in high risk DTC patients with not suppressed TSH by L-T4 treatment alone.

\section{Subjects and Methods}

\section{Selection of patients for the study}

Initially, all DTC patients followed-up during the period 2013-2014 at Ospedale Israelitico of Rome were evaluated and screened. The study group was selected by using specific inclusion and exclusion criteria in agreement with the aim of the study. Indeed, inclusion criteria were the following: a) being patient stratified as high risk according to ATA; b) being previously treated by both surgery and ${ }^{131} \mathrm{I}$; c) assuming properly (i.e. at least 1 hour before breakfast) L-T4 as the only thyroid treatment; d) having not suppressed TSH levels (i.e. $>0.1 \mathrm{mIU} / \mathrm{L}$ ); exclusion criteria were: a) having persistent cancer disease (i.e. structural and/ or biochemical) or any thyroid remnant demonstrated by post-treatment imaging; b) using drugs interfering with L-T4 absorption [11]; c) having a previously diagnosed gastrointestinal malabsorption of L-T4 [12]. Therefore, the study group encompassed 21 high risk DTC patients with non-suppressed TSH on L-T4 treatment alone; therapy of these 21 patients was shifted from L-T4 alone to L-T4 at the same dose plus liquid L-T3 at an initial fixed dose (see below). Fig. 1 illustrates the study design and selection of the study group.

\section{Institutional management of high risk DTC}

At Ospedale Israelitico of Rome the management and treatment of DTC patients follow international guidelines [1]. Patients assessed as high risk DTC are rigorously placed in TSH suppression (i.e. $<0.1$ $\mathrm{mIU} / \mathrm{L}$ ). Each patient starts L-T4 treatment just after ${ }^{131}$ I therapy. The initial L-T4 dose is tailored and normalized by $\mathrm{kg}$ body weight (i.e. $1.6-1.8 \mu \mathrm{g} / \mathrm{kg}$ ). Patient is advised to take L-T4 in fasting condition. Careful evaluation of pre-existing cardiologic status and concurrent chronic diseases and therapies are policy of the institution. Written informed consent was obtained for all participants in the study. The study was approved by Ethical Committee of Ospedale Israelitico, Rome (IT).

\section{Management of patients of the study group}

The patients of this study had been initially treated with L-T4 dose progressively increased to obtain target serum TSH [13]. In patients in whom target serum TSH was not achieved at the dose of $2.0 \mu \mathrm{g} / \mathrm{kg} /$ day or more and/or in those with a slightly lower dose showing a serum TSH above $4.0 \mathrm{mIU} / \mathrm{L}$, liothyronine treatment was added (Fig. 1). A mutual patient-physician consensus was achieved to plan this novel therapeutic approach and patients were asked to assume liquid L-T3 formulation at the initial dose of $3.55 \mu \mathrm{g}$ ( 5 drops) once a day together with L-T4. Upon re-evaluation of pharmacological thyroid homeostasis 45 days later, those subjects who continue to have not suppressed TSH were asked to increase L-T3 dose by additional 2.13-3.55 $\mu$ g (total 8/10 drops). When needed, further adjustments of L-T3 dose were tailored according to the individual biochemical assessment.

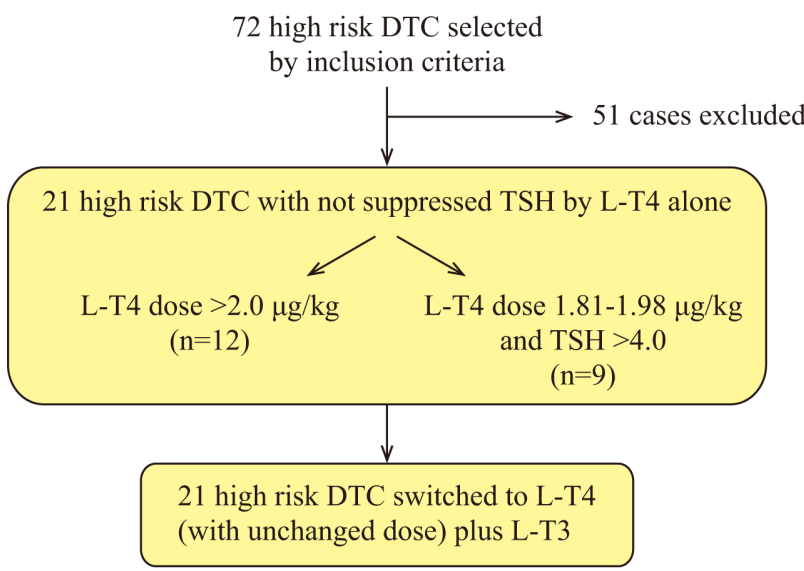

Fig. 1 Selection of patients

The study series was enrolled during the period 20132014. Inclusion and exclusion criteria are detailed in the text. 
Potential cardiologic adverse effects by thyroid hormones treatment (i.e. hypertension, tachyarrhythmia) were systematically evaluated during each patient's visit by physical examination and cardiologic visit, when appropriate.

\section{Laboratory tests}

In all subjects serum sample was drawn in the morning (i.e. between $0700 \mathrm{~h}$ and $1000 \mathrm{~h}$ ) before ingesting thyroid therapy and having breakfast. All patients underwent serum evaluation every 45 days until TSH levels were proven $<0.1 \mathrm{mIU} / \mathrm{L}$. All TSH, free- $\mathrm{T}_{3}$ and free- $\mathrm{T}_{4}$ determinations were evaluated by chemiluminescence assay.

\section{Statistical analysis}

Mean values and standard deviations were compared by paired Student's $T$-test. Correlation between L-T3 dose and body weight was analysed by linear regression. Statistical significance was set at $p<0.05$. All statistical analyses were performed using Graph Pad Prism (Graph Pad Software Inc., USA).

\section{Results}

The study group included 21 high risk DTC patients, mostly adult women, being only three patients older than 60 yr. In Table 1 are shown the anthropometric and functional characteristics of the study group. Despite L-T4 treatment alone at a mean daily dose of $2.0 \mu \mathrm{g} / \mathrm{kg}$, their TSH ranged from 0.8 to $12.0 \mathrm{mIU} / \mathrm{L}$. Following the addition of the initial dose $(3.55 \mathrm{mg} /$ day) of L-T3, TSH levels were significantly reduced in all patients and the target TSH was attained in five of them (23.8\%). In the remaining 16 patients, upon the first evaluation, the dose of L-T3 was increased by 2.13-3.55 $\mu \mathrm{g} /$ day (or 3-5 drops); at the next clinical evaluation the number of patients reaching target TSH increased to sixteen $(76.2 \%)$. Five patients required a further increase of L-T3 dose up to $14.2 \mu \mathrm{g}$ /day (or 20 drops). Finally, therefore, all 21 patients achieved the a TSH $<0.1 \mathrm{mIU} / \mathrm{L}$. Mean serum TSH value was significantly reduced at $0.05 \pm 0.04 \mathrm{mIU} / \mathrm{L}$ as compared to the initial one $3.3 \pm 3.3 \mathrm{mIU} / \mathrm{L}(p<0.001)$. The mean liquid L-T3 dose able to obtain the target TSH levels in all patients was $7.3 \pm 3.4 \mu \mathrm{g} /$ day (about 10 drops) or $0.11 \pm 0.04 \mu \mathrm{g} / \mathrm{kg} /$ day once normalized by $\mathrm{kg}$ of body weight. The individual dose varied in the 21 patients from 2.8 to $14.2 \mu \mathrm{g}$ day or 0.05 to $0.21 \mu \mathrm{g} / \mathrm{kg} /$ day, and was significantly $\left(p=0.013, \mathrm{R}^{2}=0.28\right)$ correlated with body weight (Fig. 2). On the contrary, no significant correlation was found between L-T3 dose and initial TSH value. Once TSH was suppressed, mean L-T4:L-T3 ratio was 21.3 $\pm 9.0: 1$ (ranging from 8.8 to 44.0:1).

As far as concern with serum iodothyronines, free$\mathrm{T}_{3}$ significantly $(p<0.001)$ increased up to $3.3 \pm 0.4$ $\mathrm{pg} / \mathrm{mL}$, while no significant changes of free- $\mathrm{T}_{4}$ value $(1.2 \pm 0.2 \mathrm{ng} / \mathrm{dL})$ were observed. Free- $\mathrm{T}_{3}$ increased in all cases but one (95\%). No patients showed free- $\mathrm{T}_{3}$ or free- $\mathrm{T}_{4}$ values out of the reference ranges. No cardiologic adverse events were recorded. No subjects experienced discomfort.

\section{Discussion}

Patients affected by DTC and classified at high risk of recurrence may have an improved prognosis when

Table 1 Main characteristics of patients included in the study

\begin{tabular}{lc}
\hline Patients $(\mathrm{n})$ & 21 \\
Gender (females/males) & $16 / 5$ \\
Age $(\mathrm{yr})$ & $43.1 \pm 12.0$ \\
Weight $(\mathrm{kg})$ & $64.3 \pm 7.7$ \\
Levothyroxine dose $(\mu \mathrm{g} / \mathrm{kg} /$ day $)$ & $2.0 \pm 0.2$ \\
$\mathrm{TSH}(\mathrm{mIU} / \mathrm{L})$ & $3.8 \pm 3.3$ \\
Free- $_{3}(\mathrm{pg} / \mathrm{mL})$ & $2.4 \pm 0.3$ \\
Free- $\mathrm{T}_{4}(\mathrm{ng} / \mathrm{dL})$ & $1.2 \pm 0.5$ \\
\hline
\end{tabular}

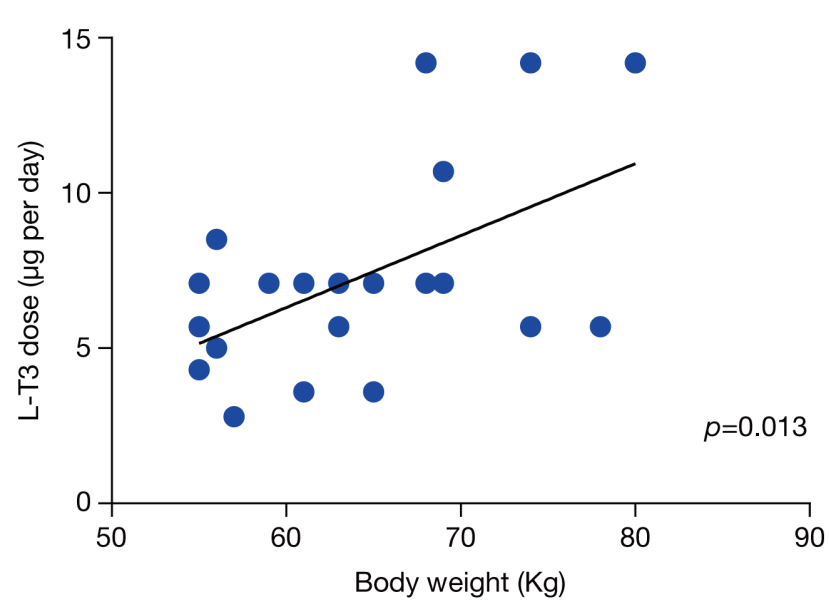

Fig. 2 Correlation between liquid L-T3 dose to suppress TSH and body weight

L-T3 represents the daily liothyronine dose added to levothyroxine which allowed to suppress TSH levels below $0.1 \mathrm{mIU} / \mathrm{L}$. 
thyrotropin levels are below $0.1 \mathrm{mIU} / \mathrm{L}$ [1]. To achieve this goal high doses of L-T4 are needed (i.e. 2.0-2.6 $\mu \mathrm{g} / \mathrm{kg} /$ day) [2, 3]. However, this high dose of L-T4 could cause adverse effects on the heart and skeleton [6]. For these reasons, a personalized regimen of thyroid hormones therapy should be recommended and the use of the more active L-T3 suggested [7]. Unfortunately, most of the studies using the combined treatment were aimed at examining mainly the quality of life and preference of patients for tablet L-T3, and quite discordant results were reported [10]. Now, liquid preparation of thyroid hormones may be more rapidly absorbed as compared with tablet formulation, which requires dissolution [14]. Direct absorption through the oral mucosa for the liquid preparation of liquid L-T4 was also proposed [15]. These advantages reported for L-T4 advised us to test the efficacy of liquid formulation of L-T3 to obtain suppressed TSH levels in patients followed-up for high risk DTC who did not show optimal suppression of TSH value during L-T4 treatment alone. Unfortunately, here we could not compare liquid $v s$. tablet L-T3 due to unavailability in Italy of low doses L-T3 (i.e. 5 or 10 or $15 \mu \mathrm{g}$ ) and because of discontinuous commercialization of the 20 $\mu \mathrm{g}$ product. As no evidence of pseudomalabsorption, food or drug interference, and gastrointestinal malabsorption have been found, their refractoriness to L-T4 may be ascribed to something else (e.g. a lower level of peripheral deiodination) [16]. The addition of a liquid L-T3 preparation to the previous dose of L-T4 stem from this hypothesis. Over a short period, by adding a mean L-T3 dose of $0.11 \mu \mathrm{g} / \mathrm{kg}$ once a day to L-T4, the desired TSH was attained in all subjects. Remarkably for clinical practice, nor side effects nor abnormal laboratory functional tests occurred, rendering overtreatment unlike [17]. In our patients, once TSH suppression was achieved a mean L-T4:L-T3 ratio of 21:1 was recorded. The latter should be quite in agreement with that suggested by ETA guidelines, in contrast with the large variability of L-T4:L-T3 ratio reported in the literature [10].

To note, regression analysis revealed a direct correlation between liothyronine dose and patients body weight, similarly to what reported for L-T4 treatment [18]. This finding is surprising since the contribution to circulating triiodothyronine, and thus its serum levels, appears to be largely affected in humans by the tissue-specific deiodination [8]. Although we have no specific data to explain this result, the supervening inhi- bition exerted by L-T3 on type 2 deiodinase (DIO2) activity which might have lowered the peripheral contribution to circulating triiodothyronine $[19,20]$ represents a suggestive hypothesis. If this assumption holds true, then serum free- $\mathrm{T}_{3}$ would reflect mainly the pharmacologic homeostasis due to the exogenous administered dose of liothyronine. In our patients treated with low dose of L-T3, serum free- $\mathrm{T}_{3}$ also increased and this may be crucial in patients lacking thyroid-derived triiodothyronine, as thyroxine in most tissues inhibits type 2 deiodinase activity and thus local triiodothyronine production. We postulate that the higher levels of serum T3, due to liothyronine administration, may strengthen the action on TSH production, in keeping with the action of $\mathrm{T} 3$ in triggering nuclear events [20]. Thus, the pituitary action of T3 may last longer than its increase in the blood [21].

The absence of gland in athyreotic patients is also an issue to be taken into account. The lack of direct triiodothyronine contribution to circulating levels in one side requires higher L-T4 amounts but on the other side the tissue distribution of DIO2 is an asymmetric one [16]. In fact, the peripheral activity of deiodinases is a tightly and tissue specific regulated process [22]. Recently, it has been shown that hypothalamic sensitivity to $\mathrm{T}_{4}$ inhibitory effect on $\mathrm{T}_{3}$ production may be lower than in other tissues. Thyroid itself may behave differently from other tissues and its removal may not account only for the mean $20 \%$ of direct $\mathrm{T}_{3}$ production but also for the response to stress of the entire deiodinase system. According with Gereben et al. [16], we may speculate that there is a gradient in tissue responsiveness to hypothyroidism and that some tissues may be slower in restoring peripheral thyroid homeostasis.

In conclusion, these preliminary data showed that liquid L-T3 can be useful to achieve optimal TSH suppression in those patients followed-up for DTC at high risk of recurrence/persistence. Indeed, the usefulness of liquid formulation of L-T3 in reaching target TSH in patients refractory to L-T4 alone has been shown. This liquid formulation allows further progressive adjustments of L-T3 with no side effects nor risk of overtreatment. Further studies to ascertain the mechanism underlying these effects are needed.

\section{Conflict of Interest}

The authors declare no conflict of interest. 


\section{References}

1. Haugen BR, Alexander EK, Bible KC, Doherty G, Mandel SJ, et al. (2016) 2015 American Thyroid Association Management Guidelines for Adult Patients with Thyroid Nodules and Differentiated Thyroid Cancer. Thyroid 26: 1-133.

2. Cooper DS, Specker B, Ho M, Sperling M, Ladenson PW, et al. (1998). Thyrotropin suppression and disease progression in patients with differentiated thyroid cancer: results from the National Thyroid Cancer Treatment Cooperative Registry. Thyroid 8: 737-744.

3. Pujol P, Daures JP, Nsakala N, Baldet L, Bringer J, et al. (1996) Degree of thyrotropin suppression as a prognostic determinant in differentiated thyroid cancer. $J$ Clin Endocrinol Metab 81: 4318-4323.

4. Jonklaas J, Bianco AC, Bauer AJ, Burman KD, Cappola AR, et al. (2014) Guidelines for the treatment of hypothyroidism: prepared by the american thyroid association task force on thyroid hormone replacement. Thyroid 24: 1670-1751.

5. Del Duca SC, Santaguida MG, Brusca N, Gatto I, Cellini M, et al. (2015) Individually-tailored thyroxine requirement in the same patients before and after thyroidectomy: a longitudinal study. Eur J Endocrinol 173: 351-357.

6. Biondi B, Cooper DS (2010) Benefits of thyrotropin suppression versus the risks of adverse effects in differentiated thyroid cancer. Thyroid 20: 135-146.

7. Biondi B, Wartofsky L (2012) Combination treatment with T4 and T3: toward personalized replacement therapy in hypothyroidism? J Clin Endocrinol Metab 97: 2256-2271.

8. Bianco AC, Salvatore D, Gereben B, Berry MJ, Larsen PR (2002) Biochemistry, cellular and molecular biology, and physiological roles of the iodothyronine selenodeiodinases. Endocr Rev 23: 38-89.

9. Gullo D, Latina A, Frasca F, Le Moli R, Pellegriti G, et al. (2011) Levothyroxine monotherapy cannot guarantee euthyroidism in all athyreotic patients. PLoS One 6: e22552.

10. Wiersinga WM, Duntas L, Fadeyev V, Nygaard B, Vanderpump MP (2012) 2012 ETA Guidelines: The Use of L-T4 + L-T3 in the Treatment of Hypothyroidism. Eur Thyroid $J$ 1: 55-71.
11. Liwanpo L, Hershman JM (2009) Conditions and drugs interfering with thyroxine absorption. Best Pract Res Clin Endocrinol Metab 23: 781-792.

12. Centanni M, Gargano L, Canettieri G, Viceconti N, Franchi A, et al. (2006) Thyroxine in goiter, Helicobacter pylori infection, and chronic gastritis. $N$ Engl J Med 354: 1787-1795.

13. Wiersinga WM (2001) Thyroid hormone replacement therapy. Horm Res 56: 74-81.

14. Yue CS, Scarsi C, Ducharme MP (2012) Pharmacokinetics and potential advantages of a new oral solution of levothyroxine vs. other available dosage forms. Arzneimittelforschung 62: 631-636.

15. Pirola I, Formenti AM, Gandossi E, Mittempergher F, Casella C, et al. (2013) Oral liquid L-thyroxine (L-t4) may be better absorbed compared to L-T4 tablets following bariatric surgery. Obes Surg 23: 1493-1496.

16. Gereben B, McAninch EA, Ribeiro MO, Bianco AC (2015) Scope and limitations of iodothyronine deiodinases in hypothyroidism. Nat Rev Endocrinol 11: 642-652.

17. Saravanan P, Siddique H, Simmons DJ, Greenwood R, Dayan CM (2007) Twenty-four hour hormone profiles of TSH, Free T3 and free T4 in hypothyroid patients on combined T3/T4 therapy. Exp Clin Endocrinol Diabetes 115: 261-267.

18. Devdhar M, Drooger R, Pehlivanova M, Singh G, Jonklaas J (2011) Levothyroxine replacement doses are affected by gender and weight, but not age. Thyroid 21: 821-827.

19. Biondi B, Wartofsky L (2014) Treatment with thyroid hormone. Endocr Rev 35: 433-512.

20. Jonklaas J, Burman KD, Wang H, Latham KR (2015) Single-dose T3 administration: kinetics and effects on biochemical and physiological parameters. Ther Drug Monit 37: 110-118.

21. Chiamolera MI, Wondisford FE (2009) Minireview: Thyrotropin-releasing hormone and the thyroid hormone feedback mechanism. Endocrinology 150: 1091-1096.

22. Bianco AC, Kim BW (2006) Deiodinases: implications of the local control of thyroid hormone action. J Clin Invest 116: 2571-2579. 